\title{
A multilevel method applied to the numerical simulation of two-dimensional incompressible flows past obstacles at high Reynolds number
}

François Bouchon, Thierry Dubois and Nicolas James

Key words: Incompressible flows, Multilevel scheme, Cut cell, Immersed boundary.

\section{Introduction}

Numerical simulation of turbulent flows in complex geometries is one of the most investigated fields in computer science in the last decades. But even though the power of supercomputers has regularely increased for many years, it has been understood that the numerical simulation of realistic flows at high Reynolds number would require too many efforts in term of memory and CPU time if one discretizes directly the Navier-Stokes equation.

In this paper we present an extension of the spectral multilevel method applied for 3D periodic turbulence and the channel flow (see [2], [3]) to the case of numerical simulation of two-dimensional flows past obstacles. In [5], these mutlilevel methods have first been adapted to the context of the finite difference discretization of the Navier-Stokes equations on staggered cartesian grids. Here, this approach is coupled with the immersed boundary (IB) method proposed in [4], which allows to handle complex geometries, and numerical results are presented and compared to "one-level" (or direct) simulations where no multilevel strategy is used. At Reynolds number 9500, comparisons are shown between the mutlilevel method on a $512^{2}$ mesh and a direct simulation on a $3072^{2}$ mesh. At Reynolds number 500 000, the multilevel method is robust, while the corresponding direct simulation (same grid, same time-step) blows up in the first time-iterations of the solution.

François Bouchon, Thierry Dubois and Nicolas James

Clermont Université, Université Blaise-Pascal, Laboratoire de Mathématiques, BP10448, F-63000 Clermont-Ferrand and CNRS, UMR 6620, Laboratoire de Mathématiques, F-63177 Aubière, email: francois.bouchon, thierry.dubois, nicolas.james@math.univ-bpclermont.fr 


\section{Description of the problem}

\subsection{The governing equations and their discretization}

We consider an incompressible viscous flow in a domain $\Omega \subset \mathbb{R}^{2}$ described by the non-dimensional Navier-Stokes equations:

$$
\begin{array}{r}
\partial_{t} u-\operatorname{Re}^{-1} \Delta u+\nabla \cdot(u \otimes u)+\nabla p=f, \\
\nabla \cdot u=0, \\
u(x, t=0)=u_{0},
\end{array}
$$

where $R e$ denotes the Reynolds number, $f$ the external volume force and $u_{0}$ the initial condition.

This system is completed with boundary conditions. The case of periodic flow will be addressed, as well as Dirichlet boundary conditions for the velocity field.

We now describe the numerical method corresponding to the case of periodic boundary conditions, the numerical treatment near the boundary is detailed in section 2.3. For the points away from $\partial \Omega$, all terms in equations (1) and (2) are discretized in space by using second-order centered finite volume schemes. The discrete unknowns are given on a staggered grid (see [7]): discrete pressure values are located at the center of mesh cells, velocity values are located at the center of edges (see Figure 1).

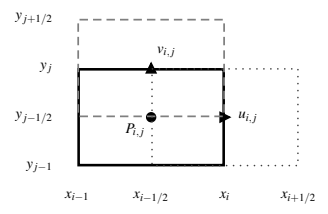

Fig. 1 Cells $K_{i-\frac{1}{2}, j-\frac{1}{2}}$ (solid), $K_{i-\frac{1}{2}, j}$ (dashed) and $K_{i, j-\frac{1}{2}}$ (dotted) and their corresponding discrete values

We define the vector $u^{k} \in \mathbb{R}^{N^{2}}$ of components $u_{i, j}^{k}$ where $N$ is the number of unknowns in each direction, and similarly, $v^{k} \in \mathbb{R}^{N^{2}}$ and $P^{k} \in \mathbb{R}^{N^{2}}$ of components $v_{i, j}^{k}$ and $P_{i, j}^{k}$ respectively, corresponding to approximate values of the unknowns at time $t^{k}=k \delta t$. We introduce $U^{k}=\left(u^{k}, v^{k}\right)$ and $F^{k}=\left(f_{u}^{k}, f_{v}^{k}\right)$ where $f_{u}^{k}$ and $f_{v}^{k}$ are vectors in $\mathbb{R}^{N^{2}}$ corresponding to the force $f$. The discrete approximation of (1) writes:

$$
\begin{gathered}
\widetilde{U}^{k+1}+\frac{2 \delta t}{3 R e} A \widetilde{U}^{k+1}=-\frac{2 \delta t}{3} G P^{k}+\frac{1}{3}\left(4 U^{k}-U^{k-1}\right) \\
+\frac{2 \delta t}{3}\left(2 F^{k}-F^{k-1}\right)-\frac{2 \delta t}{3}\left(2 N L\left(U^{k}\right)-N L\left(U^{k-1}\right)\right) \\
U^{k+1}=\widetilde{U}^{k+1}-\frac{2 \delta t}{3} G \phi^{k+1}
\end{gathered}
$$


where the matrix $A$ is the discrete approximation of the operator $-\Delta, G$ is the one of the gradient, $N L$ are the discrete nonlinear operators and $\phi^{k+1}=P^{k+1}-P^{k}$.

The discretization of the incompressibility constraint is achieved by integrating (2) over the pressure cell $K_{i-\frac{1}{2}, j-\frac{1}{2}}$, leading to

$$
D U^{k+1}=0,
$$

where $D=G^{T}$ is an approximation of the divergence operator. Combining (5) and (6), we deduce the linear system satisfied by $\phi$, namely:

$$
D G \phi^{k+1}=-\frac{3}{2 \delta t} D \widetilde{U}^{k+1}
$$

Once (7) is solved, the velocity is updated with (5). This implementation ensures that the discrete divergence of the updated velocity is zero up to the computer accuracy.

\subsection{The multilevel methodology}

The multilevel method for the MAC scheme (marker and cell) has been detailed in [5], it consists in adapting to the physical space the methods of [2] and [3].

We introduce grid operators corresponding to some spatial filtering of the velocity field. Let us consider two embedded grids (see Fig. 2): $G_{1}=\left\{\left(x_{i}, y_{j}\right), i=\right.$ $1, \ldots, N, j=1, \ldots, N\}$ and $G_{2}=\left\{\left(x_{2 i}, y_{2 j}\right), i=1, \ldots, N / 2, j=1, \ldots, N / 2\right\}$. Let $U=(U, V)$ be a velocity field defined on the fine grid $G_{1}$. As in [5], we use the restriction operator $\mathscr{R}$ which defines a filtered velocity field $\mathscr{R}(U)$ on the grid $G_{2}$, this velocity field being extended on the grid $G_{1}$ to a velocity field $\mathscr{P} \circ \mathscr{R}(U)$ via a prolongation operator $\mathscr{P}$. The velocity field $U$ can be splitted using these operators

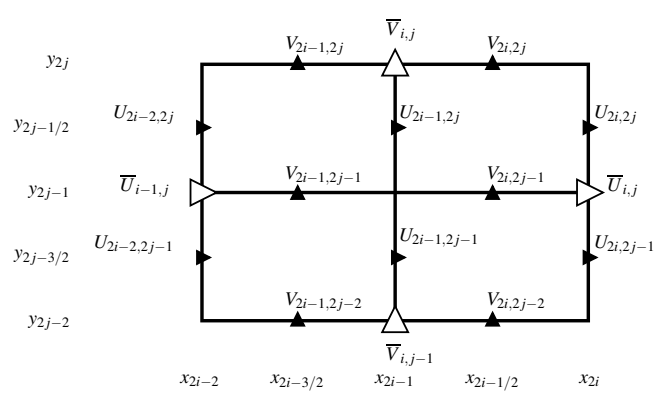

Fig. 2 Locations of the compontents of $U$ (filled style) and of $\mathscr{R}(U)$ (empty style)

$\mathscr{P}$ and $\mathscr{R}:$

$$
U=Y+Z=\mathscr{P} \circ \mathscr{R}(U)+Z
$$


It has been shown in [5] that if $D U=0$, then $D \cdot \mathscr{P} \circ \mathscr{R}(U)=0$. The component $\mathscr{P} \circ \mathscr{R}(U)$ corresponds to the large scales of the flow, and $Z$ to the smallest ones. Extending this procedure to four embedded grids, we define the following decomposition of the velocity field $U$ :

$$
U=U_{1}+U_{2}+U_{3}+U_{4}
$$

The numerical simulation consists in advancing in time $U$ as described in section 2.1 and dynamically correcting the energy contained in the smallest scales $U_{4}$ to maintain a linear discrepency of the energy spectrum in the log-log scale, following the ideas of [2] and [3] (see [5] for further details).

\subsection{A second-order immersed boundary method}

To take into accound the presence of a solid boundary embedded in $\Omega$, we use an immersed boundary technique where the unknown are placed at the middle of the part of the edges located in the fluid (see Figure 3).

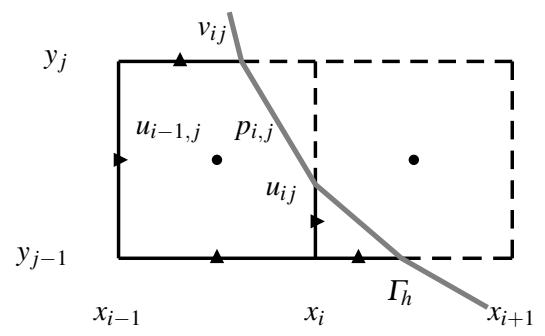

Fig. 3 Arrangement of the unknowns in cut-cells.

The Laplacien operator is then approximate at first order on these points, but due to a superconvergence property (see [8]), the numerical method is globally second order.

\section{Numerical results}

\section{1 $2 D$ periodic flows}

We first present numerical results to show the efficiency of the multilevel methodology for 2D periodic flows, by comparing on Figure 4 the spectra at Reynolds number 500000 for a direct numerical simulation on $4096^{2}$ points, and a multilevel method on $256^{2}$ points. A finer analysis in this context can be found in [5]. 


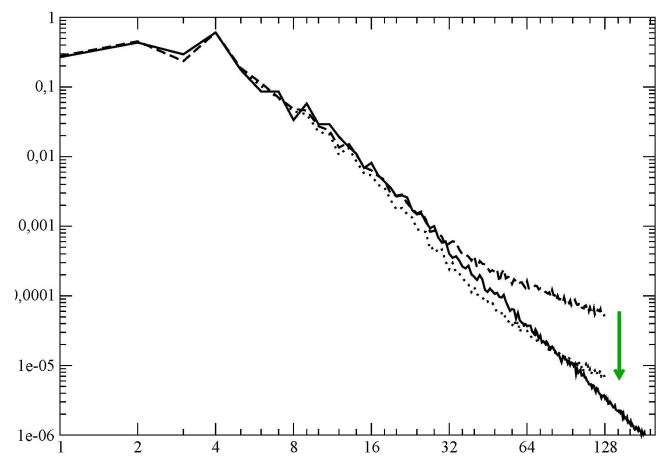

Fig. 4 Energy spectrum. DNS4096 (plain), DNS256 (long-dashed) and ML256 (dashed)

\subsection{Flow past a $2 D$ cylinder at Reynolds number 9500}

In the present application, we consider a flow in the domain $\Omega=(-5,5) \times$ $(-2.5,2.5) \backslash \mathscr{D}$, where $\mathscr{D}$ denotes the ball centered in $\mathrm{M}(0,0)$ of radius 1 . The boundary conditions are then $u=(1,0)$ on $\Sigma=\partial \Omega$ and $u=(0,0)$ on $\Gamma=\partial \mathscr{D}$. DNS have been run and results have been compared to experimental results in [1] and [6]. For $N=768$, the DNS is stable but the numerical results seem inaccurate. For $N=512$, a direct numerical simulation is unstable. For the same grid, the multi-level strategy gives numerical results close to those of the direct numerical simulation at $N=3072$ (see figure 5).

\section{Conclusion}

A multilevel method has been successively developped for two-dimensional turbulent flows at high Reynolds numbers. Comparisons with DNS on finer meshes show the efficiency of the method. A numerical simulation on a flow past obstacles at Reynolds number 500000 has also been done on a $3072^{2}$ mesh, while a DNS performed on the same mesh for the same Reynolds number leads to overflows. An analysis of this simulation and many other ones will be the subject of future works.

\section{References}

[1] R. Bouard, M. Coutanceau, The early stage of development of the wake behind an impulsively started cylinder for $40<\operatorname{Re}<10^{4}$, J. Fluid. Mech., 101, (1980) 583-607.

[2] F. Bouchon, T. Dubois, A LES model based on the spectral decay of the 


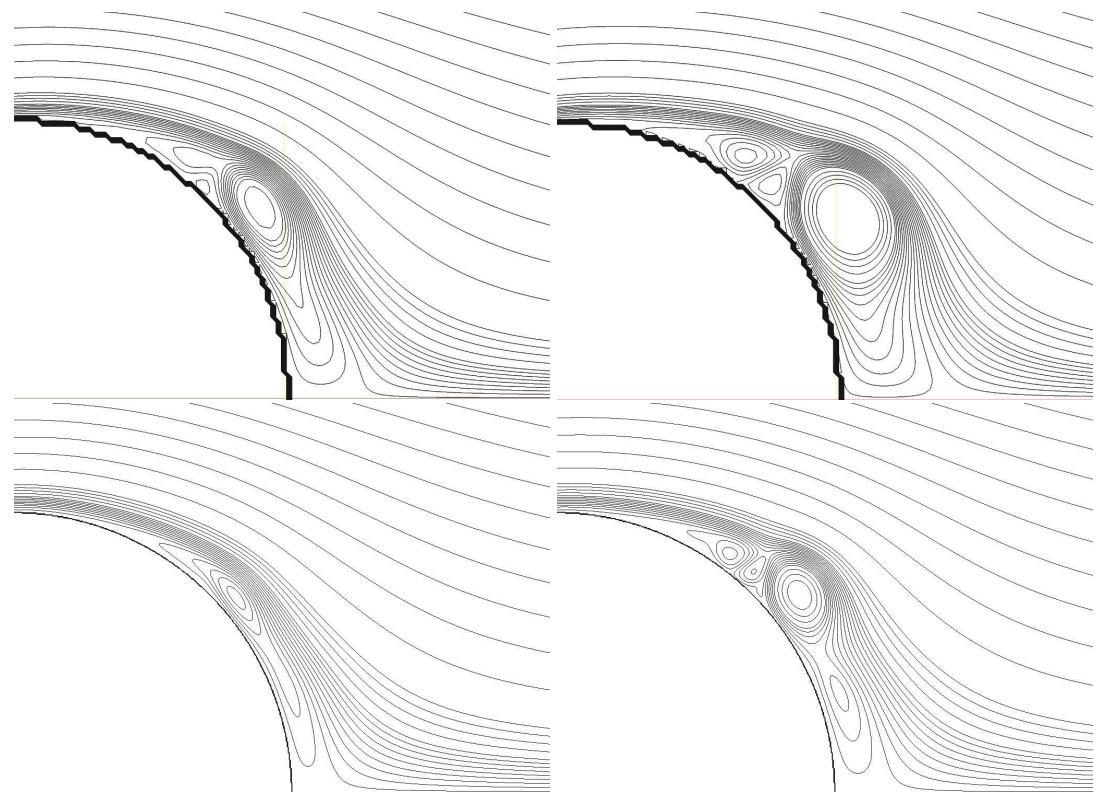

Fig. 5 Streamlines of the velocity at times 0.75 (left) and 1 (right), for the ML512 (top) and the DNS 3072 (bottom).

kinetic energy, in Advances in Turbulence VIII, CIMNE, Barcelona (2000), $527-530$.

[3] F. Bouchon, T. Dubois, A model based on incremental scales applied to LES of turbulent channel flow, in Direct and Large Eddy Simulation IV, ERCOFTAC SERIES, Kluwer Academic Publishers (2001), 97-104.

[4] F. Bouchon, T. Dubois, N. JAmes, A second-order immersed boundary method for the numerical simulation of two-dimensional incompressible viscous flows past obstacles, to appear in Computational Fluid Dynamics 2010, Proceedings of ICCFD6, St-Petersbourg, Russia, (2010) Springer.

Separation of scales on staggered grids: I. The periodic case, submitted.

[5] N. JAMES, Méthodes multi-niveaux sur grilles décalées. Application à la simulation numérique d'écoulements autour d'obstacles., Thèse de l'Université Clermont-Ferrand 2, (2009).

[6] M. Coutanceau, R. Bouard, Experimental determination of the main features of the viscous flow in the wake of a circular cylinder in uniform translation. Part 1. Steady flow, J. Fluid. Mech., 79, (1977) 231-256.

[7] F. H. HARLOW, J. E. WeLCH, Numerical calculation of time-dependent viscous incompressible flow of fluid with free surface, Phys. Fluids, 8(12), (1965) 2182-2189.

[8] N. Matsunaga, T. Yamamoto, Superconvergence of the Shortley-Weller approximation for Dirichlet problems, J. Comp. Appl. Math., 116 (2000) 263273. 